\title{
Echinococcus multilocularis in Graubünden: Verbreitung bei Füchsen und Vorkommen potentieller Zwischenwirte
}

\author{
F. Tanner'1, D. Hegglin'1, R. Thoma², G. Brosi³, P. Deplazes \\ ${ }^{1}$ Institut für Parasitologie der Universität Zürich, ${ }^{2} \mathrm{Amt}$ für Lebensmittelsicherheit und Tiergesundheit Graubünden, \\ ${ }^{3}$ Amt für Jagd und Fischerei Graubünden
}

\section{Zusammenfassung}

Die südliche Verbreitungsgrenze des europäischen Endemiegebietes von Echinococcus multilocularis verläuft entlang des Alpenkammes. Dieser endemische Übergangsbereich wurde im Kanton Graubünden kleinräumig analysiert. Dazu wurden 543 Füchse aus 10 Gebieten nördlich und 4 Gebieten südlich des Alpenhauptkammes untersucht. Parasiten wurden mit der Sedimentations- und Zähltechnik aus den Dünndarminhalten isoliert. Die mittlere Prävalenz von E. multilocularis betrug $6.4 \%$, wobei signifikante Unterschiede in den verschiedenen $\mathrm{Ge}$ bieten festgestellt wurden. In den Südtälern waren nur Füchse des Val Müstair infiziert (14.3\%). Auf der Alpennordseite schwankten die Prävalenzen zwischen 0 und $40 \%$. Mit Mageninhaltsanalysen $(n=530)$ wurde die Prädation der Füchse auf potenzielle Zwischenwirtsarten ermittelt. Nager der Gattungen Microtus/Pitymys wurden in 19.6\% der untersuchten Fuchsmägen gefunden, Clethrionomys glareolus in $8.0 \%$ und Arvicola terrestris in $0.4 \%$. Eine kleinräumige Analyse auf Basis eines $10 \times 10 \mathrm{~km}$ Rasters zeigte, dass die Prädationsrate auf die Gattungen Microtus/Pitymys signifikant mit der Prävalenz von E. multilocularis in Füchsen korreliert ist (Spearman's $r=0.51)$. Im alpinen Untersuchungsraum trat E. multilocularis somit sehr kleinräumig auf. Diese lokalen Infektionsherde persistieren möglicherweise über Jahrzehnte. Dies könnte zur Erklärung beitragen, wieso in den letzten 34 Jahren einige humane Fälle von Alveolärer Echinococcose in Gebieten des Kantons Graubünden aufgetreten sind, wo der Parasit endemisch in den Füchsen vorkommt, während in anderen, E. multilocularisfreien Gebieten des Kantons keine Fälle registriert wurden.

Schlüsselwörter: Epidemiologie, Echinococcus multilocularis, endemisches Grenzgebiet, Fuchs, Nager, Alpen

\section{Echinococcus multilocularis in Grisons: distribu- tion in foxes and presence of potential inter- mediate hosts}

The southern border of the European endemic area of Echinococcus multilocularis runs along the alpine crest. This endemic transition area was analysed in the canton Grisons on a small spatial scale. A total of 543 foxes originating from 10 areas north and 4 areas south of the main alpine divide were investigated. Parasites were isolated using the sedimentation and counting technique on intestinal contents. The mean prevalence of E. multilocularis was $6.4 \%$ with significant differences between different areas. In the southern valleys, only foxes from the Val Müstair were infected $(14.3 \%)$. On the northern side of the main alpine divide, prevalences varied between 0 and $40 \%$. The predation habits of foxes on potential intermediate hosts was investigated by means of stomach content analyses $(\mathrm{n}=530)$. Rodents of the genera Microtus/Pitymys were found in $19.6 \%$ of fox stomachs, Clethrionomys glareolus in $8.0 \%$ and Arvicola terrestris in $0.4 \%$. A small scale analysis based on a $10 \times 10 \mathrm{~km}$ grid suggested that the predation rate on the genera Microtus/Pitymys significantly correlated with the prevalence of $E$. multilocularis in foxes (Spearman's $\mathrm{r}=0.51$ ). Hence, E. multilocularis appears to occur in the alpine study area on a very small spatial scale. These local sources of infection may persist for decades. This may partly explains why, during the last 34 years, some human cases of alveolar echinococcosis occurred in areas of the Canton Grisons where the parasite is presently endemic in foxes. No such human cases have been recorded in other areas free of E. multilocularis.

Keywords: Epidemiology, Echinococcus multilocularis, endemic border, fox, rodents, Alps 


\section{Einleitung}

Die alveoläre Echinococcose (AE), verursacht durch das Larvalstadium von Echinococcus multilocularis, ist eine bedeutende Zoonose der nördlichen Hemisphäre, die unbehandelt letal verlaufen kann. Bei frühzeitiger Diagnose hingegen ist eine vollständige Heilung durch Totalresektion des Parasitengewebes möglich. Bei inoperablen Fällen kann eine über Jahrzehnte andauernde Chemotherapie das Wachstum des Parasiten einschränken (Ammann und Eckert, 1996). E. multilocularis entwickelt sich in West- und Mitteleuropa in einem Wildtier-Zyklus mit Rotfüchsen als Endwirten und verschiedenen Kleinsäuger-Arten als Zwischenwirten. Dies sind vor allem Nagetiere aus der Familie der Wühlmäuse (Arvicolidae), darunter die zwei in dieser Region wichtigsten Vertreter Microtus arvalis (Feldmaus) und Arvicola terrestris (Ostschermaus; Eckert, 1998).

Bis Ende der 80er-Jahre des letzten Jahrhunderts waren endemische Gebiete von E. multilocularis in West- und Mitteleuropa ausschliesslich aus Österreich, Deutschland, Frankreich und der Schweiz bekannt. Während der letzten 15 Jahre wurde der Parasit in mittel- und osteuropäischen Gebieten nachgewiesen, welche zuvor nicht zum Verbreitungsgebiet gezählt wurden (Eckert und Deplazes, 2004; Romig et al., 2006). Im Nordosten Europas wurden ebenfalls neue Endemiegebiete beschrieben, welche möglicherweise schon lange existierten, jedoch erst in den letzten Jahren durch systematische Untersuchungen entdeckt wurden. In anderen, heute hochendemischen Regionen, wie beispielsweise in Teilen Belgiens, Nordrhein-Westfalens oder Niedersachsen, scheint eine Ausweitung des Verbreitungsgebietes gesichert zu sein, da systematische Arbeiten hier bereits früher durchgeführt wurden (Romig, 2002). Seit Einführung der oralen Impfung gegen die Tollwut sind die Fuchspopulationen in vielen Teilen Europas, besonders auch im urbanen Siedlungsraum, angewachsen und scheinen heute grösser zu sein als je zuvor (Chautan et al., 2000; Gloor, 2002). Gleichzeitig weisen verschiedene Studien darauf hin, dass die $\mathrm{Zu}-$ nahme der Fuchspopulationen zumindest gebietsweise mit einem Anstieg der Prävalenz von E. multilocularis bei Füchsen einhergeht (Romig et al., 2006). So führten neben der Ausbreitung des Verbreitungsgebietes von E. multilocularis, die Zunahme der Fuchsbestände, die Ausbreitung der Füchse in den städtischen Lebensraum und eine Erhöhung der Prävalenz von E. multilocularis in vielen Fuchspopulationen gemeinsam dazu, dass der Infektionsdruck sowohl in ländlichen als auch in städtischen Gebieten generell deutlich zugenommen hat (Stieger et al., 2002; Eckert und Deplazes, 2004; Romig et al., 2006). Dieses Phänomen lässt uns vermuten, dass künftig auch mit mehr Fällen von $\mathrm{AE}$ beim Menschen zu rechnen ist (Deplazes et al., 2004).
In der Schweiz wurde bei grossräumigen Untersuchungen der Fuchspopulationen E. multilocularis fast flächendeckend nachgewiesen (Ewald und Eckert, 1993; Alther, 1996). Dabei unterschied sich die mittlere Prävalenz zwischen den verschiedenen Kantonen erheblich und variierte zwischen $0 \%$ (z.B. Glarus, Uri) und 53\% (Appenzell Ausserroden). Im höher gelegenen Alpenraum waren die Prävalenzen generell deutlich geringer.

Die epidemiologischen Faktoren, welche die Verbreitungsgrenzen des Endemiegebietes von E. multilocularis bestimmen, sind bisher wenig bekannt. Die Eier des Parasiten sind empfindlich gegen Trockenheit und Hitze (Veit et al., 1995). Umgekehrt konnte gezeigt werden, dass feuchte Böden das Vorkommen von $E$. multilocularis begünstigen (Staubach et al., 2001). Neben klimatischen Faktoren, so ist $\mathrm{zu}$ vermuten, spielt die Verbreitung und die Häufigkeit geeigneter Zwischenwirtsarten sowie der Prädationsdruck von Füchsen auf diese Arten eine zentrale Rolle. Diese $\mathrm{Zu}$ sammenhänge wurden jedoch bisher kaum untersucht. In Europa verläuft die südliche Verbreitungsgrenze von E. multilocularis entlang des Alpenbogens, eines sehr heterogenen Lebensraums mit mosaikartigerVerbreitung verschiedener Zwischenwirtsarten. Bisher kann derVerlauf derVerbreitungsgrenze von E. multilocularis nur sehr ungenau nachgezeichnet werden. Am Beispiel des Kantons Graubündens wurde dieser Verlauf kleinräumig untersucht und darauf geprüft, wieweit Verbreitung und Häufigkeit von E. multilocularis mit der Prädation der Füchse auf verschiedene, potentielle Zwischenwirte erklärt werden könnte.

\section{Tiere, Material und Methoden}

\section{Untersuchungsgebiet}

Das Untersuchungsgebiet, der Kanton Graubünden (7100 km², 187000 Einwohner), liegt mitten im Alpenraum und umfasst Alpentäler nördlich und südlich des Alpenhauptkamms. Das Relief erstreckt sich von einer Höhe von 260 bis 4049 Meter über Meer. Für räumliche Analysen wurde das Untersuchungsgebiet in 14 Gebiete aufgeteilt. Die Grenzen dieser Gebiete wurden entlang von Bezirks- und/oder Kreisgrenzen gezogen (Tab.1 und Abb. 1). Zehn dieser Gebiete sind nördlich und vier südlich des Alpenhauptkamms gelegen. Für kleinräumigere Auswertungen wurde zusätzlich ein $10 \times 10 \mathrm{~km}$ Raster über den Kanton gelegt.

\section{Füchse}

Zwischen November 2002 und März 2003 wurden insgesamt 543 Füchse von der Jägerschaft und Wildhut gesammelt und vom kantonalen Amt für Jagd und Fischerei zur Verfügung gestellt. Davon wurden 512 Füchse (94\%) auf der regulären Niederjagd erlegt und 


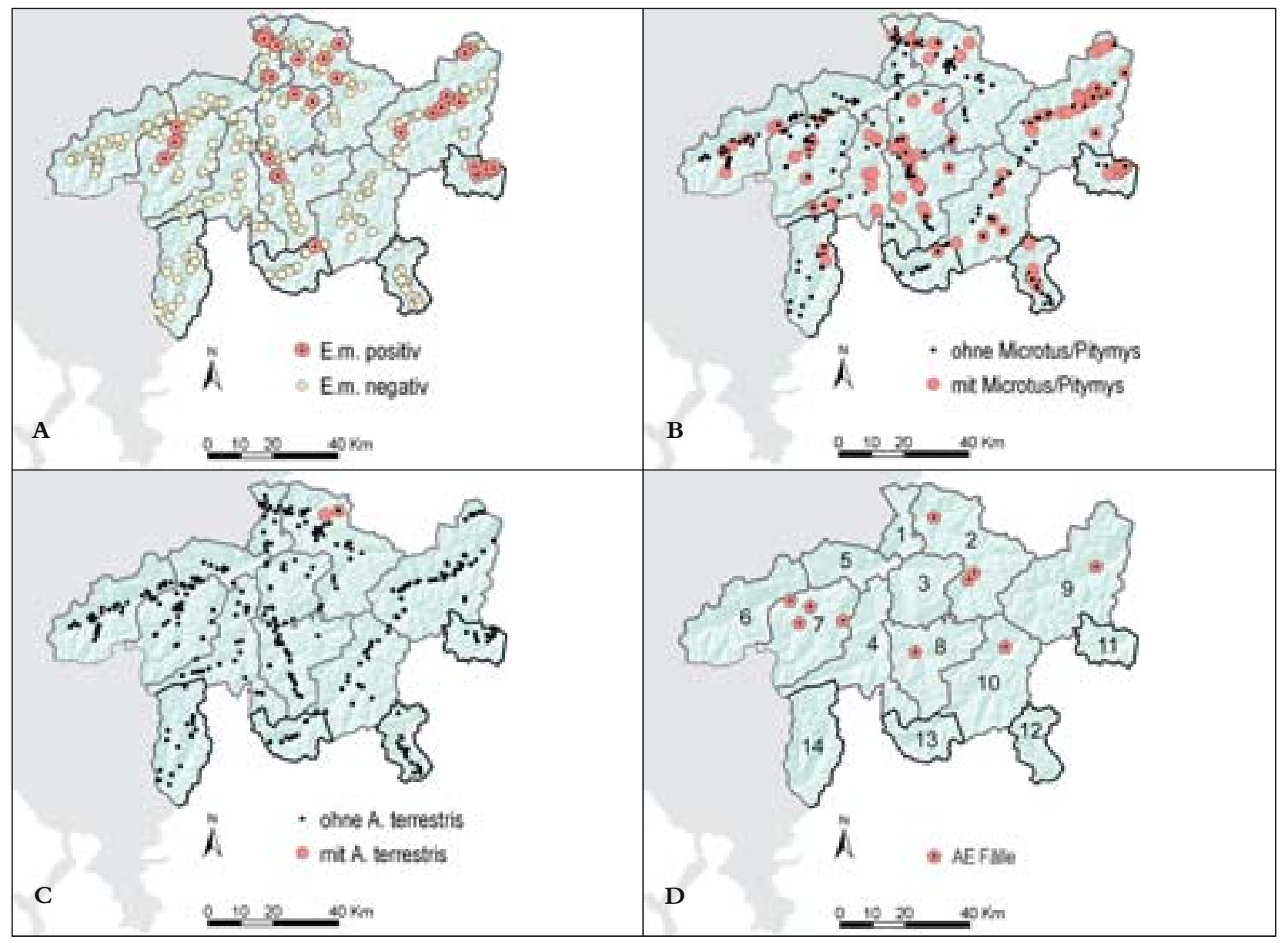

Abbildung 1: Parasitologische Untersuchungen und Mageninhaltsanalysen bei Füchsen und humane Fälle Alveolärer Echinococcose im Kanton Graubünden. Nachweise von (A) E. multilocularis in Füchsen (Darmsektionen), (B) Wühlmäusen der Gattungen Microtus/ Pitymys in Füchsmägen und (C) Arvicola terrestris in Fuchsmägen; (D) Lokalisation der medizinisch registrierten Fälle humaner Alveolärer Echinococcose zwischen 1970 und 2004 (Gloor 1988 und Prof.Ammann, persönliche Mitteilung, Eckert et al.1995) und Gebietsnummern (s. Tab. 1). Graue Linien: Gebietsgrenzen nördlich des Alpenkammes; schwarze Linien: Gebietsgrenzen südlich des Alpenkammes.

Tabelle 1: Einteilung des Kantons Graubünden in 14 Gebiete. Die Grenzen der Teilgebiete wurden nach administrativen und geographischen Kriterien festgelegt.

\section{Name (Gebietsnummer) ${ }^{\star}$}

\section{Gebiete der Alpennordseite:}

Landquart (1)

Prättigau/Davos (2)

Albula-Nord/Plessur (3)

Hinterrhein (4)

Surselva-Nord/Imboden (5)

Surselva-West (6)

Surselva-Süd (7)

Albula-Süd (8)

Engiadina bassa (9)

Engiadin' ota (10)

\section{Gebiete der Alpensüdseite:}

Val Müstair (11)

Val Posciavo (12)

Val Bregaglia (13)

Val Mesolcina/Calanca (14)

\section{Administrative Einheiten ${ }^{\star}$}

Bezirk Landquart

Bezirk Prättigau/Davos

Kreise Alvaschein und Belfort und Bezirk Plessur

Bezirk Hinterrhein

Kreis Ilanz nördlich des Vorderrheins und Bezirk Imboden

Kreis Disentis und Kreis Ruis nördlich des Vorderrheins

Kreise Lugnez und Safien und Kreise Ruis und Ilanz südlich des Vorderrheins Bezirk Albula ohne Kreise Alvaschein und Belfort

Bezirk Inn ohne Kreis Val Müstair

Bezirk Maloja ohne Kreis Bergell

\section{Kreis Val Müstair}

Bezirk Bernina

Kreis Bergell

Bezirk Moesa

* Der Grenzverlauf der Gebiete ist anhand der Gebietsnummern aus Abbildung 1D ersichtlich.

** Administrative Einteilung Kanton Graubünden (1.Jan. 2003):

http://www.gis.gr.ch/karten/bezirke_kreise_gemeinden/pdf/einteilung_in_bezirke_kreise_gemeinden.pdf 
31 Füchse (6\%) als Fallwild aufgefunden. Die Sektion der Füchse erfolgte durch das Kantonale Veterinäramt in Chur, welches Dünndärme und Mägen der Füchse zur weiteren Untersuchung an das Institut für Parasitologie der Universität Zürich weiterleitete. Für die epidemiologischen Auswertungen wurden von allen Füchsen Datum und Erlegungsort protokolliert. Das Alter der untersuchten Füchse betrug mindestens 7 Monate.

\section{Parasitologische Untersuchung}

Die Untersuchung der Fuchsdärme erfolgte gemäss den von Deplazes und Eckert (1996) beschriebenen Sicherheitsvorschriften. Nach dem Tiefgefrieren während 5 Tagen bei $-80^{\circ} \mathrm{C}$, folgte die Untersuchung der Fuchsdärme mit der Sedimentations- und Zähltechnik auf intestinale Helminthen (Hofer et al., 2000).

\section{Mageninhaltsanalyse}

Vor der Analyse wurden alle Mägen eine Woche bei $-80^{\circ} \mathrm{C}$ gelagert, um allenfalls vorhandene Eier von $E$. multilocularis abzutöten, und bis zur Weiterverwendung bei $-20^{\circ} \mathrm{C}$ aufbewahrt. Die Aufarbeitung der Mageninhalte erfolgte nach Capt und Stalder (1988). Dazu wurden die Mägen aufgeschnitten und in einem Haushaltssieb (Lochgrösse $2 \mathrm{~mm}$ ) unter sanftem Wasserstrahl gereinigt, bis das abfliessende Wasser klar war. Die Nahrungsresten liessen sich in einem wassergefüllten Becken nach Nahrungskategorien sortieren. Haare und Gewebe von Nagern sowie Haare von anderen Säugern wurden in 70\%-Alkohol fixiert. Die Artbestimmung erfolgte durch die Analyse von Deckhaaren, die in reines Paraffin eingebettet und mit einem Microtom in $6 \mu \mathrm{m}$ dünne Querschnitte aufgeschnitten wurden. Die Bestimmung erfolgte bei $400-$ facher Vergrösserung mit den Schlüsseln von Day (1966) und von Teerink (1991). Falls Haare anhand des Querschnittes nicht identifizierbar waren, wurde deren Medulla und Cuticula beurteilt. Dies erfolgte auf einem Objektträger mit einer dünnen Schicht farblosen Nagellacks bei 100- bis 400-facherVergrösserung. Für die Begutachtung der Cuticula konnte das Haar vorsichtig vom Objektträger entfernt werden. Der verbleibende Abdruck der Cuticula wurde anhand des Schlüssels von Teerink (1991) identifiziert.

\section{Dichteindizes der Wühlmäuse Microtus/Pitymys}

Zwischen dem 5. und 13. September 2004 wurden Dichteindizes für die Populationsdichte von Wühlmäusen bestimmt. Dazu wurden drei Gebiete mit unterschiedlicher E. multilocularis Prävalenzen bei Füchsen ausgewählt: Auf der Alpennordseite ein Kreis mit hoher Prävalenz (Kreis Lugnez) und drei benachbarte Kreise ohne E. multilocularis Nachweise (Kreise Disen- tis, Ruis, Ilanz); auf der Alpensüdseite das Val Müstair (Kreis Val Müstair) mit hoher Prävalenz. Für die Bestimmung der Dichteindizes wurde ein leicht modifiziertes Verfahren nach Quéré (2000) angewandt: Transekte von 100 bis 300 Schritten Länge und einem Meter Breite wurden festgelegt, und für jedes Transekt wurde alle 5 Schritte protokolliert, ob Spuren von kleinen Wühlmausarten (Eingangslöcher, oberirdische Gangsysteme) gefunden werden konnten. $\mathrm{Zu}-$ sätzlich wurde protokolliert, ob in den Gangsystemen Losungen der Nager zu sehen waren. Es war nicht möglich, die Gangsysteme von Microtus- und Pitymysarten auseinander zu halten, weshalb ein Dichteindex für die Gruppe Microtus/Pitymys gemeinsam errechnet wurde.

\section{Statistische Auswertungen}

Da E. multilocularis auf der Alpensüdseite bisher nur vereinzelt nachgewiesen wurde, erfolgte die Auswertung in zwei räumlichen Skalen: Mit dem Mann-Whitney UTest wurde in einem ersten Schritt überprüft, ob sich die Prävalenzen und Prädationsraten auf verschiedene $\mathrm{Na}$ gerarten zwischen den Gebieten der Alpennord- und der Alpensüdseite unterscheiden. In einem zweiten Schritt wurde für die Alpennord- und Alpensüdseite separat getestet, ob sich kleinräumig zwischen den einzelnen Gebieten signifikante Unterschiede ergeben. Dazu kam der Actus-Randomisation Test (George F. Estabrook, New Hampshire, USA) zur Anwendung, ein Test der randomisierte Kontingenztabellen generiert und für die einzelnen Zellen die Wahrscheinlichkeit einer Abweichung von den Erwartungswerten ausgibt (Estabrook und Estabrook, 1989).

Mit dem Spearman-Korrelationskoeffizent wurde die Korrelation zwischen der E. multilocularis-Prävalenz bei Füchsen und der Prädation von Füchsen auf verschiedene Nagerarten in den 14 Gebieten überprüft. Dazu wurde für jedes Gebiet die Prädationsrate auf Wühlmausartige (Arvicolidae gesamthaft sowie Microtus/Pitymys und C. glareolus gesondert), echte Mäuse (Muridae) und Spitzmäuse (Soricidae) separat errechnet. Um auch kleinräumigere Korrelationen zu erfassen, wurden die entsprechenden Prävalenzen und Prädationsraten auch für jede Zelle eines $10 \times 10 \mathrm{~km}$ Rasters, das über das Untersuchungsgebiet gelegt wurde, bestimmt. Um den Effekt der grossenVarianz kleiner Stichproben zu reduzieren wurden nur Rasterquadrate mit $\geqq 10$ untersuchten Füchsen berücksichtigt ( $N=21$ Rasterquadrate). Unterschiede der Dichteindizes für Mictrotus/ Pitymys zwischen den untersuchten Gebieten wurden mit dem Kruskal-Wallis Test statistisch abgesichert. Für binominaleVariablen wurde das exakte 95\% Konfidenzintervall nach Clopper und Person (1934) berechnet. Die statistischen Analysen wurden mit den Softwarepaketen SPSS-PC Vers. 10 (Norusis, 2000) und ACTUS2 (Estabrook und Estabrook, 1989) durchgefuihrt. 


\section{Ergebnisse}

\section{Verbreitung von E. multilocularis in Füchsen}

Von 543 untersuchten Fuchsdärmen waren 35 mit E. multilocularis befallen (mittlere Prävalenz: 6.4\%). Der grösste Teil dieser Füchse zeigte einen geringen bis mittleren Befall (<100 Individuen: 40\%; 1001000 Individuen: 40\%). Nur 20\% der infizierten Füchse waren mit mehr als 1000 Echinococcen befallen. Nebst dem Befall mit E. multilocularis waren die Füchse mit Mesocestoides (65\%), Hakenwürmer (Ancylostomatiden, 63.5\%), Spulwürmer (Ascaridae, $59.9 \%$ ) und Taenia spp. (44.0\%) befallen.

In einem der 4 Gebiete der Alpensüdseite und in 7 der 10 Gebiete der Alpennordseite wurde E. multilocularis nachgewiesen (Tab. 2). Die höchste Prävalenz wurde im Alpennordseits gelegenen Bezirk Landquart registriert (40\%), gefolgt vom alpensüdseits gelegenen Val Müstair (14.3\%). Zwischen den Nord- und Südtälern gesamthaft ergab sich kein signifikanter Unterschied (Mann-Whitney U =13.5, p > 0.05). Jedoch bestehen zwischen den einzelnen Gebieten signifikante regionale Unterschiede (Alpennordseite: $\mathrm{p}<0.001$; Alpensüdseite: $\mathrm{p}<0.05)$.

\section{Prädation der Füchse auf verschiedene Zwischenwirte}

Die Mageninhaltsanalyse erfolgte bei 530 Füchsen; 26 Mägen waren leer (4.9\%). In 175 Mägen (33.0\%) waren Reste von Mäusen in Form von Haaren, Gewebe oder auch ganzen Mäusen erkennbar. Da anhand der Haaranalyse die Arten Microtus arvalis, Microtus agrestis und Pitymys subterraneus nicht zu unterscheiden sind, wurden diese Reste zur Nagergruppe Microtus/Pitymys zusammengefasst. Die Fuchsmägen enthielten in abnehmender Häufigkeit Microtus/Pitymys (19.6\%), Clethrionomys glareolus (8.0\%) und Arvicola terrestris $(0.4 \%)$. Zusätzlich befanden sich in $3.5 \%$ der Mägen Apodemus, in 5.4\% Soricidae und in $0.4 \%$ Mus musculus.

In Tabelle 2 sind die Prädationsraten auf die als Zwischenwirte bedeutsamen Wühlmäuse (Microtus/Pity$m y s, A$. terrestris., C. glareolus) für jedes Gebiet separat dargestellt. Bei allen drei Gruppen ergab sich kein signifikanter Unterschied zwischen der Alpensüd- und der Alpennordseite (Microtus/Pitymys: Mann-Whitney $\mathrm{U}=17.5, \mathrm{p}>0.5 ;$ C. glareolus: $\mathrm{U}=8.0, \mathrm{p}>0.05 ;$ A terrestris: $\mathrm{U}=18, \mathrm{p}>0.05)$. A. terrestris fanden wir jedoch nur in 2 Füchsen aus St. Antönien (Prättigau). C. gla-

Tabelle 2: E. multocularis-Prävalenzen in Füchsen in Graubünden, und Anteil von Fuchsmägen mit Resten von Arvicola terrestris, Microtus / Pitymys spp. und Clethrionomys glareolus, sowie zwischen 1970-2004 aufgetretene Fälle Alveolärer Echinococcose.

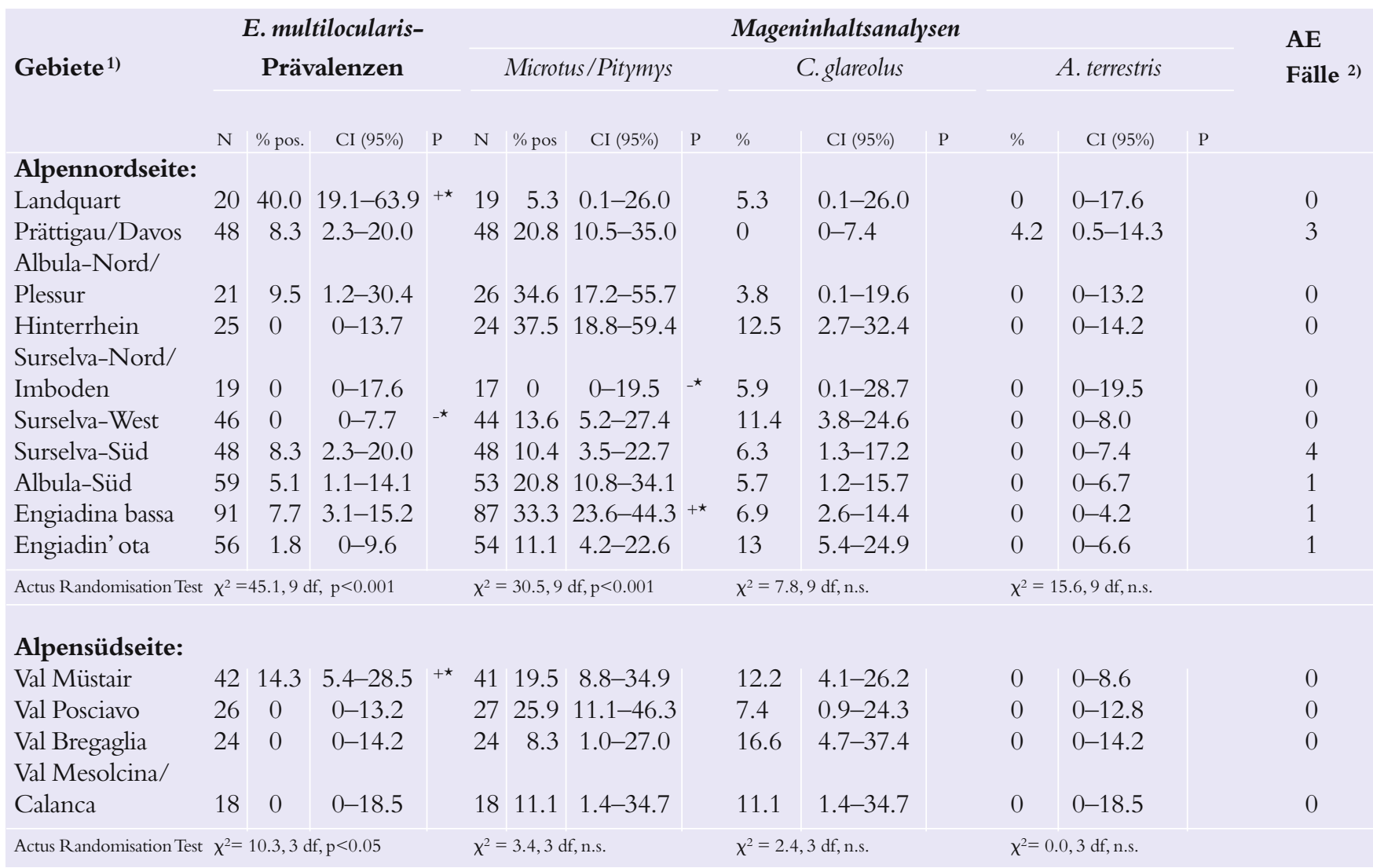

Die Signifikanz der räumlichen Unterschiede wurde für die Regionen der Alpennordseite und der Alpensüdseite separat mit dem Actus-Randomisation-Test errechnet. Felder mit positiver (+) und negativer (-) Abweichung vom Erwartungswert sind mit dem Symbol * $(p<0.05)$ gekennzeichnet. 1) wurden nach Bezirken und Kreisen eingeteilt, siehe Tabelle 1 und Abbildung 2

2) aus (Eckert et al., 1995; Gloor, 1988) sowie Prof. R. Ammann, persönliche Mitteilung 
Tabelle 3: Räumliche Korrelation der Prävalenz von E. multilocularis in Füchsen mit dem Anteil verschiedener Nager, die in Fuchsmägen nachgewiesen wurden. Die Korrelationen wurden auf Basis der 14 Gebiete $(N=14)$ und einem $10 \times 10 \mathrm{~km}$ Raster (nur Rasterquadrate mit $>=10$ Füchsen wurden berücksichtigt; $N=21$ ) errechnet. $r=$ Spearman's Korrelationtskoeffizent, $p=$ Irrtumswahrscheinlichkeit.

\begin{tabular}{|c|c|c|c|c|}
\hline & \multicolumn{2}{|c|}{14 Gebiete } & \multicolumn{2}{|c|}{21 Rasterquadrate } \\
\hline & $\mathbf{r}$ & $\mathbf{p}$ & $\mathbf{r}$ & $\mathbf{p}$ \\
\hline Arvicolidae & 0.021 & 0.944 & 0.335 & 0.138 \\
\hline - Microtus/Pitymys & 0.046 & 0.876 & 0.510 & $0.018 \star$ \\
\hline - C. glareolus & -0.531 & 0.051 & -0.299 & 0.187 \\
\hline Muridae & 0.490 & 0.075 & 0.184 & 0.424 \\
\hline Soricidae & -0.122 & 0.678 & 0.260 & 0.255 \\
\hline
\end{tabular}

$\star P<0.05$

reolus und Microtus/Pitymys wurden in Fuchsmägen aus 13 von 14 Gebieten nachgewiesen. In der Region Prättigau/Davos konnten keine C. glareolus und in der Region Surselva-Nord/Imboden keine Microtus/Pitymys nachgewiesen werden. Dabei zeigten sich für C. glareolus sowohl auf der Alpensüd- als auch auf der Alpennordseite keine bedeutenden Unterschiede zwischen den verschiedenen Gebieten ( $p>0.05$, Tab. 2). Hingegen waren die Unterschiede bei der Gruppe Microtus/Pitymys zwischen den Gebieten der Alpennordseite hoch signifikant $(\mathrm{p}<0.001$, Tab. 2). Der Anteil der Mägen die Microtus/Pitymys enthielten, bewegte sich hier zwischen $0 \%$ und 37.5\%.Auf der Basis eines $10 \times 10 \mathrm{~km}$ Rasters, nicht aber auf Basis der 14 Gebiete, liess sich eine signifikante Korrelation zwischen der Prädationsrate von Füchsen auf Microtus/ Pitymys und der Prävalenz von E. multilocularis in Füchsen nachweisen $(r=0.51, p=0.018$, Tab. 3).

\section{Nagerdichte und E. multilocularis-Prävalenz bei Füchsen}

Insgesamt wurden die Dichteindizes für Nager der Gattungen Microtus/Pitymys auf 60 Transekten (Kreis Val Müstair 22, Kreis Lugnez 20, Kreise Disentis/ Ruis/Ilanz 18) erhoben. Die E. multilocularis-Prävalenz unterschied sich signifikant zwischen diesen drei Gebieten und war hoch im Kreis Val Müstair (14.3\%, Tab. 2) und im Kreis Lugnez (17.4\%, CI 5-38.8\%). In den Kreisen Disentis, Ruis und Ilanz wurde kein infizierter Fuchs gefunden (0\%, CI $0-3.2 \%)$. Sowohl die Häufigkeit von Losungen von Microtus/ Pitymys als auch die Häufigkeit von Spuren dieser Artengruppe (oberirdische Gangsysteme und Eingangslöcher) war am geringsten in diesen Kreisen (Abb. 2).Allerdings konnte der Unterschied zwischen den drei Gebieten nur für die Häufigkeit oberirdischer Gangsysteme und Eingangslöcher statistisch abgesichert werden (Abb. 2, Kruskal-Wallis Test: $\chi^{2}=$ 6.6, 2 df, $\mathrm{p}<0.05)$. In allen drei Gebieten wurden nie Spuren von $A$. terrestris gefunden.

\section{Diskussion}

Die mittlere Prävalenz von E. multilocularis beim Fuchs ist im Alpenraum deutlich niedriger (vorliegende Studie: 6.4\%) als im Schweizer Mittelland.Aufgrund unserer Ergebnisse können wir annehmen, dass dies primär auf eine mosaikartige Verteilung von Echinococcus-freien Gebieten und Gebieten mit fokal erhöhtem Auftreten des Parasiten zurückgeführt werden kann. So wurde in unserer Studie eine maximale Prävalenz von $40 \%$ nachgewiesen, ein ähnlich hoher Wert wie er in verschiedenen Kantonen des Schweizer Mittellands gefunden wurde (Ewald und Eckert, 1993; Alther, 1996). Umgekehrt konnte in 6 der 14 untersuchten Gebiete gar keine E. multilocularis-Infektion entdeckt werden. Dieses kleinräumige Verteilungsmuster von E. multilocularis in einem geographisch heterogenen Lebensraum bietet die Möglichkeit, den Einfluss verschiedener Faktoren auf die Dynamik des Lebenszyklus von E. multilocularis zu untersuchen.

Südlich des Alpenhauptkamms wurden bis Ende der 90er-Jahre nur vereinzelt infizierte Füchse nachgewiesen, immer in geringer Distanz zu den Alpennordtälern (Ewald und Eckert, 1993; Manfredi et al., 2002). Für infizierte Füchse aus dem italienischen Grenzgebiet zu Österreich wurde vermutet, dass es sich um einzelne, von der Alpennordseite abgewanderte Füchse handeln könnte, und dass sich so der Lebenszyklus von E. multilocularis auf der Alpensüdseite (noch) nicht etabliert hat (Manfredi et al., 2002).Auch im Nordtessin wurde seit der erstmaligen Untersuchungen von Ewald keine spätere Ausweitung nach Süden festgestellt (Alther, 1996). Allerdings wurde in der italienischen Provinz Bozen erstmals in einem grösseren Gebiet E. multilocularis südlich des Alpenhauptkamms festgestellt. Von 171 untersuchten

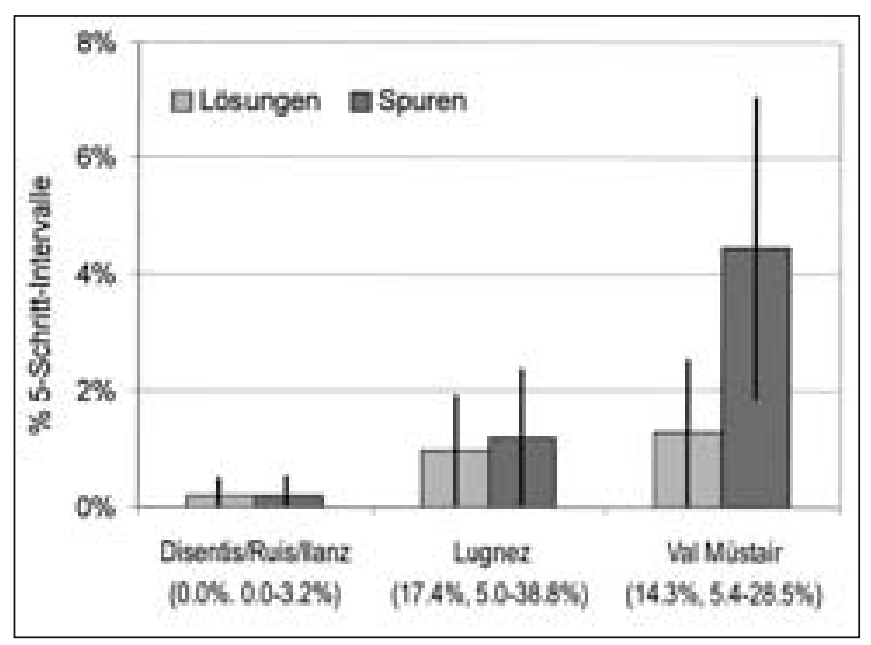

Abbildung 2:Anteil an 5-Schritt-Intervallen mit Losungen und Spuren (Eingangslöcher, oberirdische Gangsysteme) von Nagern der Gruppe Pitymys/Microtus (schwarze Linie 95\% CI). In Klammer sind die jeweiligen Echinococcus multilocularis-Prävalenzen und die zugehörigen 95\%-CI Intervalle angegeben. 
Losungen gesammelter Füchse waren 22 PCR-positiv (12.9\%). Allerdings gelang der direkte ParasitenNachweis mittels Darmsektionen nur bei zwei dieser Füchse (Casulli et al., 2005). In unserer Studie konnte nun erstmals E. multilocularis direkt mittels Darmsektionen in einer erheblichen Prävalenz auf der Alpensüdseite nachgewiesen werden. In 6 von 42 Füchsen des Val Müstair (14.3\%) parasitierten zwischen 5 und 792 E. multilocularis Würmer. Gleichzeitig konnten in 19.5\% der Fuchsmägen Nager der Gruppe Microtus/ Pitymys erfasst werden, und auf den Wiesenflächen waren häufiger Spuren von Microtus/Pitymys nachweisbar, als in Gebieten mit niedriger Prävalenz. Dabei handelt es sich mit hoher Wahrscheinlichkeit vorwiegend um $M$. arvalis, da $M$. agrestis in diesem Tal bisher nicht beschrieben wurde und die untersuchten Wiesenflächen nicht typisch für die vorkommenden Pitymysarten sind (Tab. 2, Hausser, 1995 und pers. Mitteilung J.P. Müller). Obwohl ein direkter Nachweis infizierter Zwischenwirte von E. multilocuaris bisher in den Alpensüdtälern fehlt, weisen unsere Resultate darauf hin, dass der Lebenszyklus von $E$. multilocularis sehr wahrscheinlich auch südlich des Alpenhauptkamms etabliert ist. Wie weit dies auf eine generelle Zunahme und Ausbreitung dieses Parasiten zurück zu führen ist, lässt sich nicht beurteilen, da umfangreichere Erhebungen aus früheren Jahren fehlen. Möglicherweise hat sich E. multiocularis erst in den letzten Jahren in das Val Müstair von Italien oder vom Engadin her ausgebreitet. Die neusten Daten aus Italien (Casulli et al., 2005) lassen jedoch vermuten, dass das Endemiegebiet des Val Müstair heute mit dem italienischen Endemiegebiet (Provinz Bozen) verbunden ist.

Die fokal erheblichen Prävalenzen von E. multilocularis südlich des Alpenhauptkamms zeigen, dass nicht geographische Barrieren, sondern andere Faktoren, wie klimatische oder ökologische Parameter, die südliche Verbreitungsgrenze im Alpenraum festzulegen scheinen. Im urbanen Siedlungsraum, wo die Streifgebiete von Füchsen besonders klein sind (Gloor, 2002) wurde gezeigt, dass die Prävalenz bei Füchsen innerhalb von wenigen 100 Metern bedeutend ändern kann (Hegglin et al., 2003). Dabei wird die Prävalenz massgeblich vom Angebot geeigneter Zwischenwirte bestimmt (Deplazes et al., 2004). Am Stadtrand von Zürich, wo ausreichend Wiesenflächen Lebensraum für $A$. terrestris und $M$. arvalis bieten, haben Füchse häufiger diese beiden Wühlmausarten erbeutet und waren entsprechend häufiger von $E$. multilocularis befallen als in zentralen Stadtteilen. Auch in dieser Untersuchung wurden kleinräumig erhebliche Unterschiede gefunden: Die Prävalenzen von E. multilocularis zwischen den Füchsen verschiedener Gebiete Graubündens variieren beträchtlich. Dabei ist keine graduelle Abnahme der Prävalenz gegen Süden hin ersichtlich, sondern vielmehr treten infizierte Füchse geklumpt auf (Tab. 2 und Abb. 1). Diese heterogene Verbreitung von E. multilocularis scheint auch im alpinen Lebensraum wesentlich von einer unterschiedlichen Prädationsrate auf wenige, geeignete Leit-Zwischenwirtsarten bestimmt zu sein. Die Magenanalysen zeigen, dass die als bedeutende Zwischenwirtsart bekannte $A$. terrestris nur in Nordgraubünden möglicherweise eine gewisse Rolle als Zwischenwirt spielt, wo vereinzelt Exemplare dieser Art in Füchsmägen nachgewiesen werden konnten. C. glareolus wurden mit einer Ausnahme (Prättigau/Davos) in allen Gebieten in den Fuchsmägen nachgewiesen. Die höchsten Prädationsraten wurden jedoch in Gebieten gefunden, in denen $E$. multilocularis noch nie gefunden wurde (Tab. 2). Entsprechend war zwischen der Prädationsrate auf diese Art und der E. multilocularis-Prävalenz bei Füchsen eine in der Tendenz negative Korrelation zu finden (Tab. 3). Auch wenn C. glareolus als Zwischenwirt in der Schweiz erfasst wurde (Stieger et al., 2002), gibt es bisher keine verlässlichen Daten, dass ihr eine LeitZwischenwirtsfunktion zukommt, die den Zyklus unabhängig von anderen Arten aufrechterhalten kann. Die signifikante Korrelation zwischen der Prävalenz von E. multilocularis in Füchsen und der Prädation von Mäusen der Gattungen Microtus/Pitymys, nicht aber der Prädation von A.terrestris und C. glareolus, weist darauf hin, dass in Graubünden der Lebenszyklus von E. multilocularis massgebend von $M$. arvalis bestimmt ist. Die wichtige Zwischenwirtsfunktion dieser Art wurde auch für andere Gebiete West- und Mitteleuropas beschrieben (Bonnin et al., 1986; Delattre et al., 1990; Gottstein et al., 2001). Die PitymysArten und Microtus agrestis treten in Graubünden allgemein weniger häufig auf (Müller, pers. Mitt.) und wurden bisher in keiner uns bekannten Studie als bedeutende Zwischenwirte beschrieben. Jedoch sollten künftig umfangreichere, vergleichende Prävalenzstudien zu diesen Zwischenwirtsarten durchgeführt werden, um dieses Resultat schlüssig abzusichern. Die Korrelation zwischen der Prädationsrate auf die Gattungen Microtus/Pitymys und der Prävalenz von E. multilocularis in Füchsen war nur kleinräumig auf der Basis eines $10 \times 10 \mathrm{~km}$ Rasters signifikant, und nicht auf Basis der 14 grossflächigeren Gebiete. Dies weist darauf hin, dass auch im alpinen Raum der Zyklus sehr kleinräumig von einem lokal ausreichend grossem Angebot geeigneter Zwischenwirte abzuhängen scheint. Über die Stabilität des Zyklus von E. multilocularis im Alpenraum ist noch wenig bekannt. Möglicherweise haben solche lokalen Foci über lange Zeit Bestand. Dies könnte erklären, wieso einige humane Fälle von $\mathrm{AE}$ über den langen Zeitraum zwischen 1970-2004, wie im Gebiet Surselva-Süd und Prättigau/Davos sehr nahe beieinander aufgetreten sind (Abb. 1). Dem gegenüber wurden in Gebieten Sur- 
selva-West, Surselva-Nord/Imboden und Hinterrhein, in denen E. multilocularis noch nie nachgewiesen wurde (Ewald und Eckert, 1993; aktuelle Studie), bisher auch keine Fälle von $\mathrm{AE}$ registriert.

Neue Aspekte über die Dynamik des Vorkommens von E. multilocularis in Endemiegrenzgebieten wie dem des Kantons Graubünden erhoffen wir über molekularepidemiologische Untersuchungen der verschiedenen Echinococcus-Populationen mittels Mikrosatellitenanalysen (Bart et al., 2006) sowie über Langzeitstudien, die auch temporale Parameter über das Vorkommen von E. multilocularis in unserem Untersuchungsgebiet berücksichtigen werden.

\section{Echinococcus multilocularis dans les Grisons: Répartition chez les renards et présence d'hôtes intermédiaires potentiels}

La limite méridionale du domaine endémique d'Echinococcus multilocularis sui la crête des Alpes. Cette zone de transition a été analysée sur la base de 14 sécteurs dans tout le canton des Grisons. A cet effet, 543 renards provenant de 10 secteurs au nord et de 4 au sud des Alpes ont été examinés. Les parasites ont été isolés du contenu de l'intestin grêle par une technique de sédimentation et de comptage. La prévalence d'E. multilocularis était de $6,4 \%$ avec des différences significatives selon les localisations. Dans les vallées méridionales, seuls les renards du Val Münster étaient infectés (14,3\%). Au nord des Alpes la prévalence varie de 0 à $40 \%$. En analysant le contenu stomacal $(\mathrm{N}=530)$, on a déterminé la consommation d'hôtes intermédiaires potentiels par les renards. Des rongeurs des genres Microtus/Pitymys ont été trouvé dans 19,6\% des estomacs, Clethrionomys glareolus dans $8 \%$ et Arvicola terrestris dans $0,4 \%$. Une analyse fine à l'aide d'un quadrillage à maille de $10 \mathrm{~km}$ par $10 \mathrm{~km}$ a montré que le taux de prédation sur les espèces Microtus/ Pitymys étaient corrélé de façon significative avec la présence E. multilocularis chez les renards. Dans la zone alpine examinée, E. multilocularis était présent de façon très localisée. Ces foyers d'infections locaux persistent vraisemblablement durant des décennies. Cela pourrait contribuer à expliquer que, dans les 34 dernières années, quelques cas humains d'echinococcose alvéolaire soient apparus dans des parties des Grisons où le parasite existe de façon endémique chez les renards alors que dans d'autres parties indemnes d' E. multilocularis aucun cas n'a été enregistré.

\section{Dank}

Herzlichen Dank geht an alle Jäger, Jagdaufseher und Wildhüter für ihr Engagement. Speziellen Dank geht insbesondere an Lucia Kohler und Claude Fischer für Datenerhebung- und Auswertung, sowie an Jürg-Paul Müller und Thomas Briner vom Bündner Naturmuseum und an Simon Capt vom Centre Suisse de Cartographie de la Faune. Diese Arbeit stellt die Dissertation von Frau Flurina Tanner dar.

\section{Echinococcus multilocularis nei Grigioni: distribuzione nelle volpi e presenza di potenziali ospiti intermedi}

Il limite meridionale dell'area endemica europea dell' Echinococcus multilocularis si situa lungo le Alpi. Questa zona di transizione endemica è stata esaminata in scala ridotta del Canton Grigioni. Sono stati analizzate 543 volpi di 10 zone settentrionali e di 4 meridionali della zona alpina. I parassiti sono stati isolati dal contenuto dell'intestino tenue con tecniche di sedimentazione e di conteggio. La prevalenza media di E. multilocularis ammonta al $6.4 \%$, con differenze significative nelle diverse zone. Nelle valli meridionale solo le volpi provenienti dalla Val Monastero erano infettate (14,3\%). Nella parte nord delle Alpi le prevalenze variavano tra 0 e $40 \%$. Con le analisi del contenuto dello stomaco $(n=530)$ è stata rilevata le predazione delle volpi su potenziali specie di ospiti intermedi. Roditori del genere Microtus/Pitymys sono stati ritrovati nello stomaco delle volpi analizzate nel $19,6 \%$, il Clethrionomys glareolus nell' $8,0 \%$ e l'Arvicola terrestris nello $0,4 \%$ dei casi. Un'analisi in scala ridotta sulla base di quadrati di $10 \times 10 \mathrm{~km}$ ha mostrato che la tasso significativa di predazione dei generi Microtus/Pitymys è correlata con la prevalenza di E. multilocularis nelle volpi (Spearman's $\mathrm{r}=0,51)$. Nelle zone alpine esaminate l'E. multilocularis ha una distribuzione geografica no omogenea. Probabilmente questi ceppi infettivi locali persistono da più decenni. Questo potrebbe spiegare il perché, negli ultimi 34 anni, alcune forme umane di Echinococcosi alveolare si sono presentate nel Canton Grigioni dove il parassita sussiste in forma endemica nelle volpi mentre in altre zone libere da E. multilocularis del Cantone non sono stati registrati casi. 


\section{Literatur}

Alther P.: Beitrag zur Epidemiologie und Diagnose der Echinococcus multilocularis-Infektion bei Endwirten. Dissertation, Universität Zürich, 1996.

Ammann R.W., Eckert J.: Cestodes.Echinococcus. Gastroenterol. Clin. N. 1996, 25:655-689.

Bart J.M., Knapp J., Gottstein B., El-Garch F., Giraudoux P., Glowatzki M.L., Berthoud H., Maillard S., Piarroux R.: EmsB, a tandem repeated multi-loci microsatellite, new tool to investigate the genetic diversity of Echinococcus multilocularis. Infect. Genet. Evol. 2006.

Bonnin J.L., Delattre P., Artois M., Pascal M., Aubert M.F, Petavy A.F: Contribution à la connaissance des hôtes intermédiares d'Echinococcus multilocularis dans le nord-est de la France. Description des lésions trouvées chez 3 espèces de rongeurs naturellement infectés. Ann. Parasitol. Hum. Comp. 1986, 61:235-243.

Capt S., Stalder H.W.: Untersuchung zur Habitatnutzung von Rotfüchsen (Vulpes vulpes L.) im Schweizerischen Alpenraum. Dissertation, Universität Bern, 1988.

Casulli A., Manfredi M.T., La Rosa G., Di Cerbo A.R., Dinkel A., Romig T., Deplazes P., Genchi C., Pozio E.: Echinococcus multilocularis in red foxes (Vulpes vulpes) of the Italian Alpine region: is there a focus of autochthonous transmission? Int. J. Parasitol. 2005, 35:1079-1083.

Chautan M., Pontier D., Artois M.: Role of rabies in recent demographic changes in red fox (Vulpes vulpes) populations in Europe. Mammalia 2000, 64:391-410.

Clopper C.J., Pearson E.S. : The use of confidence or fiducial limits illustrated in the case of the binomial. Biometrika 1934, 26:404-413.

Day M.G.: Identification of hair and feather, remains in the gut and faeces of stoats and weasels. J. Zool. (London). 1966, 148:201-217.

Delattre P., Giraudoux P., Quere J.P.: Epidemiological Consequences of the Receptivity of a New Intermediate Host of Echinococcus-Multilocularis and of the Space-Time Localization of the Infected Rodents. Cr. Acad. Sci. - Vie. 1990, 310:339-344.

Deplazes P., Eckert J.: Diagnosis of the Echinococcus multilocularis infection in final hosts.Appl. Parasitol. 1996,37:245-52.

Deplazes P., Hegglin D., Gloor S., Romig T.: Wilderness in the city: the urbanization of Echinococcus multilocularis. Trends $\mathrm{Pa}$ rasitol. 2004, 20:77-84.

Eckert J.: Alveolar Echinococcosis (Echinococcus multilocularis) and other forms of echinococcosis (Echinococcus vogeli and Echinococcus oligarthrus). In: Zoonoses. Hrsg. S.R. Palmer, L. Soulsby and D.I.H. Simpson, Oxford Medical Publications, 1998, 689-716.

Eckert J., Deplazes P.: Biological, epidemiological and clinical aspects of echinococcosis: a zoonosis of increasing concern. Clin. Microbiol. Rev. 2004, 17:107-135.
Eckert J., Jacquier P., Baumann D., Raeber P.A.: Echinokokkose des Menschen in der Schweiz, 1984-1992. Schweiz. Med.Wschr. 1995, 125:1989-1998.

Estabrook C.B., Estabrook G.F:Actus: a solution to the problem of small samples in the analysis of two-way contingency tables. Hist. Method. 1989, 82:5-8.

Ewald D. : Prävalenz von Echinococcus multilocularis bei Rotfüchsen (Vulpes vulpes L.) in der Nord-, Ost- und Südschweiz sowie im Fürstentum Liechtenstein. Dissertation, Universität Zürich, 1993.

Gloor B.: Echinokokkose beim Menschen in der Schweiz 1970-1983. Dissertation, Universität Zürich, 1988.

Gloor S.: The rise of urban foxes (Vulpes vulpes) in Switzerland and ecological and parasitological aspects of a population in the recently colonised city of Zurich. Dissertation, Universität Zürich, 2002.

Gottstein B., Saucy F., Deplazes P., Reichen J., Demierre G., Busato A., Zuercher C., Pugin P.: Is high prevalence of Echinococcus multilocularis in wild and domestic animals associated with disease incidence in humans? Emerg. Infect. Dis. 2001, 7:408-412.

Hausser J.: Säugetiere der Schweiz:Verbreitung - Biologie Ökologie. Birkhäuser Verlag, Basel, Schweizerische Gesellschaft für Wildtierbiologie, 1995, 501.

Hegglin D., Ward P.I., Deplazes P.: Anthelmintic baiting of foxes against urban contamination with Echinococcus multilocularis. Emerg. Infect. Dis. 2003, 9:1266-1272.

Hofer S., Gloor S., Muller U., Mathis A., Hegglin D., Deplazes P.: High prevalence of Echinococcus multilocularis in urban red foxes (Vulpes vulpes) and voles (Arvicola terrestris) in the city of Zurich, Switzerland. Parasitology 2000, 120:135-142.

Manfredi G., Deplazes P., Trevisiol K., Fraquelli C.: Echinococcus multilocularis infection in red foxes in Italy.Vet. Rec.2002, 150:757.

Norusis M.J.: SPSS 10.0 guide to data analysis. First edition. Prentice Hall, Upper Saddle River, New Jersey, USA. 2000.

Quéré J.-P., Raoul F., Giraudoux P., Delattre P.: An index method of estimating relative population densities of the common vole (Microtus arvalis) at landscape scale. Rev. Ecol. TerreVie. 2000, 55:25-32.

Romig T.: Spread of Echinococcus multilocularis in Europe? Cestode Zoonoses: Echinococcosis and Cysticercosis, P. Craig and Z. Pawlowski, Eds., IOS Press, Amsterdam, Netherlands, 2002, 65-80.

Romig T., Dinkel A., Mackenstedt U.: The present situation of echinococcosis in Europe. Parasitol. Int. 2006, 55 Suppl: 187-191. 
Staubach C., Thulke H.H., Tackmann K., Hugh-Jones M., Conraths FJ.: Geographic information system-aided analysis of factors associated with the spatial distribution of Echinococcus multilocularis infections of foxes. Am. J. Trop. Med. Hyg. 2001, 65:943-948.

Stieger C., Hegglin D., Schwarzenbach G., Mathis A., Deplazes P. Spatial and temporal aspects of urban transmission of Echinococcus multilocularis. Parasitology 2002, 124:631-640.
Teerink B.J.: Hair of west european mammals: Atlas and Identification Key, Cambridge University press, 1991, 232.

Veit P., Bilger B., Schad V., Schafer J., Frank W., Lucius R.: Influence of environmental factors on the infectivity of Echinococcus multilocularis eggs. Parasitology 1995, 110:79-86.

\section{Korrespondenzadresse}

Peter Deplazes, Institut für Parasitologie, Universität Zürich, Winterthurerstrasse 266a, 8057 Zürich E-Mail: deplazesp@access.unizh.ch,Fax:+41446358907

Manuskripteingang: 7. Juni 2006

Angenommen: 13.Juni 2006 